\title{
Decoherence of Two-Level Systems Can Be Very Different from Brownian Particles
}

\author{
B. L. $\mathrm{Hu}^{*}$ \\ Department of Physics, University of Maryland, College Park, Maryland 20742 \\ (umdpp 02-038, Feb. 27, 2002. To appear in Chaos, Solitons, Fractals)
}

\begin{abstract}
In quantum computation, it is of paramount importance to locate the parameter space where maximal coherence can be preserved in the qubit system. Considerable insight in environment-induced decoherence has been gained in the last decade from detailed studies using the quantum Brownian motion (QBM) models. A number of respectable authors have applied these insights derived from QBM models to two level systems interacting with a field. Their conclusions based on this particular type of qubit coupling to the environment had led to the general belief that 2LS are easily decohered. In a recent paper [1], we debunk such a myth and caution indiscriminate application of the QBM model of decoherence to arbitrary two level systems. We point out that at least for a two-level atom (2LA)- electromagnetic field (EMF) system alone, as used in the atom cavity prototypes of quantum computers, the decoherence time is rather long, comparable to the relaxation time. In the standard Hamiltonian of the 2LA, the dominant interaction is the $\hat{\sigma}_{ \pm}$type of coupling between the two levels (what constitutes the qubit) and the field, not the $\hat{\sigma}_{z}$ type assumed in most previous discussions of qubit decoherence, which shows the QBM behavior. Depending on the coupling the field can act as a resonator (in an atom cavity) or as a bath (in QBM) and produce very different decoherent behavior. Our conclusion is based on a new
\end{abstract}

*hub@physics.umd.edu 
exact master equation we derived at zero temperature which generalizes the text-book ones restricted by the Born-Markov approximation. Indeed many cavity experiments testify to the correctness of these results, and that the 2LA-EMF system maintaining its coherence in sufficiently long duration is the reason why experimentalists can manipulate them to show interesting quantum coherence effects.

\section{Introduction}

A two-level system (2LS) interacting with an electromagnetic field (EMF) has proven to be a very useful model for a wide range of problems from atomic-

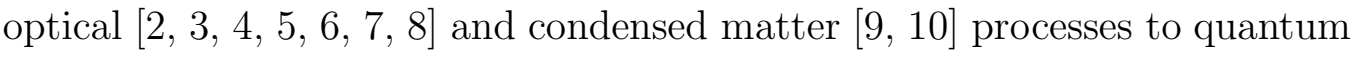
computation [11. For the latter application stringent limits in maintaining the coherence of the 2LS (called qubits) are required. This prompted us to revisit the theoretical structure of the 2LS-EMF system, paying special attention to its relaxation and decoherence properties 112, 13, 14, 15, 16, 17, 18, 19.

Environment-induced decoherence [20] has been studied extensively in recent years primarily based on models of quantum Brownian motion (QBM) [21, 22, 23, 24, 25, 26, 27, 28] for the interaction of a simple harmonic oscillator (Brownian particle) with a harmonic oscillator bath (HOB) at a finite temperature, leading to a reasonably good understanding of its characteristic features. Decoherence of a 2LS in an EMF has been studied by a number of authors, notably [12, 13, 17], and their dissipative and decoherent behavior are reported to be similar to that of a QBM in a harmonic oscillator bath. They described the progression in three stages - quiescent, vacuum fluctuation-dominated and thermal fluctuation-dominated, separated by the cutoff frequency and the thermal de Broglie frequency (wavelength), which are characteristic QBM features [22, 24, 25, 29, 30, 31, 32]. A recent comprehensive review of environment-induced decoherence can be found in [33].

Our findings, based on the standard 2LA-EMF model [2], are in stark disagreement from that reported in the literature. From solutions of the exact master equation for the reduced density matrix obtained recently by Charis Anastopoulos and the author [1] capable of treating non-Markovian dynamics at zero temperature, the decoherence time of the 2LA is found to be close to the relaxation time. In many circumstances these times are rather 
long - indeed, that is why observations of many optical coherence phenomena are possible. This result is, in first appearance, rather counter-intuitive, and different from all previous findings. However, as will be explained here, the 'intuition' one forms about the nature of dissipation and decoherence are hitherto largely based on the QBM model. It is quite general, yet by no means universal. This convenient extrapolation could have influenced the choice of model in previous authors' investigation and the readers' impression of decoherence for a 2LS. Our findings show that such a commonly invoked intuition for QBM in a HOB fails to apply to that of a two-level atom (2LA) interacting with an electromagnetic field (EMF). Our conclusion shows the need for extra caution in accepting categorical depiction of decoherence: One needs to identify the correct type of coupling of the 2LS with its environment and consider different decoherent mechanisms at work in a realistic experiment.

\section{Decoherence in $Q B M$}

The folklore that decoherence of a Brownian oscillator proceeds in a very short time (typically $10^{-40}$ of the dissipation time) is really based on high temperature ohmic bath conditions. This is widely known because it is the only technically simple case studied in detail [22, 20]. It is by no means universal. Intuitively, the bath needs to have many degrees of freedom, preferably acting independently of each other so incooperatively that the phase information in the system will be dispersed to the largest extent amongst the many bath degrees of freedom and affords little chance or takes inordinately long time to be revived or reconstituted (recoherence, see, e.g., [34]). Long decoherence times can appear in cases of low temperature, supraohmic bath, as was first pointed out by $\mathrm{Hu}$, Paz and Zhang [25]in analyzing decoherence behavior of QBM based on their exact non-Markovian master (HPZ) equation for general environments. (For details based on numerical and analytical solutions of the HPZ equation, see [29, 28]). The almost opposite picture (of very long decoherence time) is exemplified by two coupled subsystems where no coarse-graining is introduced, such as the 'dressed atom' description [7] made possible because of the coherence established between a 2LA and a single resonant mode in the cavity. (Collapse and revival of the Rabi nutation are distinct features of quantum coherence [2].) This shows that both the coupling and the nature of the environment are important 
factors affecting the decoherence of the system.

We also want to point out that equating a 2LS with QBM could be as mistaken as ignoring the basic difference between dissipative and mixing systems. To the extent that QBM exemplifies the former, spin echo phenomena (e.g, Chap. 3 [5])is an example of the latter. Plasma waves showing Landau damping in Vlasov dynamics arising from a mean field approximation is another example [35, 36] of the latter - the 'damping' is really a misnomer. Just as in the spin echo phenomena, the basic physics in this case is not dissipation in the Boltzmann sense, but statistical mixing [37]. We will see that the statistical mechanical properties of a $2 \mathrm{LA}-\mathrm{EMF}$ system is closer to the latter than the QBM, and reflects in their different decoherence nature.

Coherence in the $2 L S$

For the 2LA-EMF system, one clear distinction between an EM field and a system of harmonic oscillators acting as bath is that the field (coupled to a detector) has an intrinsic spectral density function, which cannot be chosen arbitrarily. For example, it has been shown 26] that a conformal scalar field in two dimensions coupled to a monopole detector has an Ohmic character while in four dimensions it is supraohmic . Barone and Caldeira 38 showed that the spectral density function for EM fields with momentum coupling to an oscillator detector is supraohmic. These density functions would show very different decoherent behavior from the high temperature Ohmic HOB case which molds the common impression of decoherence.

But the most important distinction from QBM is that the 2LA couples with the EMF in the discrete number basis for the field, unlike the continuous amplitude basis in the QBM. This fact (which is true in the rotating wave and dipole approximation) implies that the 2LS plus EMF system is a resonant one. Hence even though the EM field has just as many (in fact, a large number of) modes as the HOB, only a very small fraction of them in a narrow range of the resonance frequency are efficiently coupled to the atom. This is the root cause for the very different qualitative behavior between the QBM and the 2LS as far as decoherence is concerned.

We see that in a $2 \mathrm{LA}$, for purely radiative decay the decay time $T_{1}$ of the inversion is half the decay time $T_{2}$ of the polarization. There is no large order of magnitude differences between dissipation and decoherence time. In fact it is perhaps inappropriate to talk about dissipation for a 2LA-EMF system 
because the conditions for a bath to actuate such a process is lacking. The transition from excited to ground state is closer in nature to relaxation (with relaxation time constant $\Gamma$ ) than dissipation. In a cavity where excitation of the atom from the field (absorption)balances with emission, it is more appropriate to refer to the resonant state of the atom-field as a coherent system. In these scenarios the distinction between QBM and 2LA cannot be clearer.

\section{Difference between $Q B M$ and $2 L S$}

So what led earlier authors to make the claim that 2LS decoheres easily? We think the confusion arises when the picture of QBM dissipation and decoherence is grafted on the 2LA-EMF system indiscriminately. If the field which acts as the environment is a phonon field (from ion vibrations, see, e.g., [18]), if there is atomic collisions in a cavity [8] or if the cavity walls are imperfect, some of these decoherent agents could follow the QBM pattern as reported by many authors. Such sources can be important for some setups.

Quantitatively, the model for the 2LS used by most authors for the discussion of decoherence inspired by QBM type of behavior has the atom in a $\hat{\sigma}_{z}$ state (the diagonal Pauli matrix) coupled to the field mode operators $\hat{b}^{\dagger}, \hat{b}$. This type of coupling term (call it $\hat{\sigma}_{z}$ type for convenience) commutes with the Hamiltonian of the system, and admits a diagonalization in the eigenbasis of the Hamiltonian. The field is coupled to the atom as a whole, not to the two level system (qubit), which is at the heart of quantum computation. By contrast the standard model for 2LA-EMF which we studied has a $\hat{\sigma}_{ \pm}$coupling to the field modes which controls the two- level activity of the atom. This coupling considered in the standard model is indispensable, i.e., it cannot be removed from the two-level atom as it defines it and will be present in any realistic situation. Where then could the QBM type of interaction enter in the 2LA?

If the EM field is the only environment present, we can still ask if a QBM type of coupling term with the EM field would appear, and if yes, how strong would its effect be? A useful way to compare the relative importance of these two types of coupling would be to seek out the source where they stem from.

Recall that the standard model is derived under the dipole and rotating wave approximations. In the next section we will show that the $\hat{\sigma}_{z}$ type of coupling appears only in the next order expansion after the dipole approximation. Since these are good approximations for a large class of atomic 
states when the atom is nonrelativistic, the contribution from the QBM type of coupling used in [12, 13, 14, 17] should be negligible in a 2LA-EMF system and its ensuing decoherent effect insignificant. In this sense the EM field does not in leading order of approximation act like a bath in the QBM way, and coherence in a 2LA-EMF system is quite well preserved (excepting other processes, e.g. [16, 15]).

Decoherent behavior of the 2LA-EMF system we are reporting on here is based on an analysis of the full (non-Markovian) dynamics in the cases of a free quantum field and a cavity field at zero temperature from solutions of an exact master equations derived by Anastopoulos and $\mathrm{Hu}$ [1]. They used the influence functional method [21] to take into account the full backreaction of the field on the atom, while adopting Grassmannian variables for the 2LA and the coherent state representation for the EMF.

\section{The Model}

Our model for atom-field interaction is the standard one (see Appendix A of [1] for details) [2, 3, 5] 巴. The total Hamiltonian for a (stationary) atom interacting with a quantum electromagnetic field (EMF) under the dipole, rotating wave (RW) and two-level (2L) approximation is given by

$$
\hat{H}=\hbar \omega_{0} \hat{S}_{z}+\hbar \sum_{\mathbf{k}}\left[\omega_{\mathbf{k}} \hat{b}_{\mathbf{k}}^{\dagger} \hat{b}_{\mathbf{k}}+\left(g_{\mathbf{k}} S_{+} \hat{b}_{\mathbf{k}}+\bar{g}_{\mathbf{k}} S_{-} \hat{b}_{\mathbf{k}}^{\dagger}\right)\right]
$$

where $\hat{b}_{\mathbf{k}}^{\dagger}, \hat{b}_{\mathbf{k}}$ are the creation and annihilation operators for the kth normal mode with frequency $\omega_{\mathbf{k}}$ of the electromagnetic field (thus for the field vacuum $\hat{b}_{\mathbf{k}}|0\rangle=0,\left[\hat{b}_{\mathbf{k}}, \hat{b}_{\mathbf{k}^{\prime}}^{\dagger}\right]=\delta_{\mathbf{k}, \mathbf{k}^{\prime}}$, for all $\mathbf{k}$.), and $\omega_{0}=\omega_{21}$ is the frequency between the two levels. Here

$$
\hat{S}_{z}=\frac{1}{2} \hat{\sigma}_{z}, \hat{S}_{ \pm}=\hat{\sigma}_{ \pm} \equiv \frac{1}{2}\left(\hat{\sigma}_{x} \pm i \hat{\sigma}_{y}\right)
$$

where $\hat{\sigma}_{x, y, z}$ are the standard $2 \mathrm{x} 2$ Pauli matrices with $\hat{\sigma}_{z}=\operatorname{diag}(1,-1)$, etc. The coupling constant $g_{\mathbf{k}} \equiv d_{21 \mathbf{k}} f_{\mathbf{k}}(\mathbf{X})$ where

$$
d_{i j \mathbf{k}} \equiv-\frac{i \omega_{i j}}{\sqrt{2 \hbar \omega_{\mathbf{k}} \epsilon_{0} V}} \mathbf{d}_{i j} \cdot \hat{\mathbf{e}}_{\mathbf{k} \sigma}
$$

\footnotetext{
${ }^{1}$ Our Hamiltonian is given in the so-called minimal coupling (MC) as different from the multipolar coupling (MP) [7], which may be more relevant to atoms in a cavity because the explicit Coulomb interaction between the atom and its image charge is removed.
} 
and $\mathbf{d}_{i j} \equiv e \int \bar{\phi}_{i} \mathbf{x} \phi_{j} d^{3} x$ is the dipole matrix element between the eigenfunctions $\phi_{i}$ of the electron-field system, $\hat{\mathbf{e}}_{\mathbf{k} \sigma}$ is the unit polarization vector ( $\sigma=1,2$ are the two polarizations), and $f_{\mathbf{k}}(\mathbf{x})$ is the spatial mode functions of the vector potential of the electromagnetic field (in free space, $f_{\mathbf{k}}(\mathbf{x})=e^{-i \mathbf{k} \cdot \mathbf{x}}$, $V$ is the volume of space.). Under the dipole approximation $f_{\mathbf{k}}$ is evaluated at the position of the atom $\mathbf{X}$. Since $\mathbf{d}_{i j}=\overline{\mathbf{d}}_{j i}, \bar{d}_{i j \mathbf{k}}=d_{j i \mathbf{k}}$, we will choose a mode function representation such that $g_{\mathbf{k}}$ is real. When only one mode in the EM field is considered, this is the Jaynes-Cummings model.

To see how this could possibly be related to the $\hat{\sigma}_{z}$ type of coupling with Hamiltonian (used by e.g., [13, 17] for the study of decoherence in 2LS)

$$
\hat{H}=\hbar \omega_{0} \hat{S}_{z}+\hbar \sum_{\mathbf{k}}\left[\omega_{\mathbf{k}} \hat{b}_{\mathbf{k}}^{\dagger} \hat{b}_{\mathbf{k}}+\hbar \hat{\sigma}_{z}\left(\bar{g}_{\mathbf{k}} \hat{b}_{\mathbf{k}}+g_{\mathbf{k}} \hat{b}_{\mathbf{k}}^{\dagger}\right)\right]
$$

we examine the next term after the dipole approximation. This has a contribution to $g_{i j \mathbf{k}}$ even when $i=j$. This is equal to

$$
g_{i i \mathbf{k}}=c_{\mathbf{k}} \mathbf{k} \cdot \mathbf{q}_{i}
$$

where

$$
\mathbf{q}_{i}=\sum_{\sigma} \int \bar{\phi}_{i} \delta \mathbf{x}\left(\mathbf{p} \cdot \hat{\mathbf{e}}_{\mathbf{k} \sigma}\right) \phi_{i} d x^{3}
$$

and $c_{k}$ is a constant given by

$$
c_{\mathbf{k}}=-\frac{e}{m}\left(2 \hbar \omega_{\mathbf{k}} \epsilon_{0} V\right)^{-1 / 2}
$$

It generates an additional coupling term

$$
\sum_{\mathbf{k}} \hat{\sigma}_{z}\left(g_{1 \mathbf{k}} b_{\mathbf{k}}+\bar{g}_{1 \mathbf{k}} b_{\mathbf{k}}^{\dagger}\right)+1\left(g_{2 \mathbf{k}} b_{\mathbf{k}}+\bar{g}_{2 \mathbf{k}} b_{\mathbf{k}}^{\dagger}\right)
$$

where

$$
g_{1 \mathbf{k}}=g_{11 \mathbf{k}}-g_{22 \mathbf{k}}, \quad g_{2 \mathbf{k}}=g_{11 \mathbf{k}}+g_{22 \mathbf{k}}
$$

This gives the lowest order $\hat{\sigma}_{z}$ type of coupling in a 2LA -EMF system. The ratio of the coupling $g_{1 \mathbf{k}}$ of the $\hat{\sigma}_{z}$ type in (2.4) to the dipole coupling $g_{\mathbf{k}}$ in (2.1) is

$$
\left|g_{1 \mathbf{k}} / g_{\mathbf{k}}\right|=\left|\frac{\mathbf{k}\left(\mathbf{q}_{1}-\mathbf{q}_{2}\right)}{m \omega_{\mathbf{k}} d_{12}}\right| \leq \frac{\omega_{\mathbf{k}}\left|\mathbf{q}_{1}-\mathbf{q}_{2}\right|}{m \omega_{\mathbf{k}} d_{12}}
$$

Thus the $\hat{\sigma}_{z}$ type of coupling generated from the 2LA- EMF interaction will be significant only for very high frequencies $\omega_{\mathbf{k}}$ of the EM field, a point intuitively clear from the meaning of the dipole approximation. 


\section{The Master Equation}

We use the open system approach and the influence functional formalism to derive a master equation for the 2LA with backreaction of the field treated self-consistently. This involves deriving the influence functional and the evolution operator for the reduced density matrix of the 2LA. For convenience we use Grassmannian variables for treating fermions, and the coherent state representation for the field. The coherent state of the combined atom-field system is

$$
|\{z\}, \eta\rangle=|\{z\}\rangle \times|\eta\rangle
$$

where $|z\rangle, z$ a complex number, denotes the EM field coherent states and $|\eta\rangle, \eta$ a Grassmannian or anticommuting number, denotes the electron coherent state. We assume initially that the density matrix of the total system+environment is factorizable $\hat{\rho}(0)=\hat{\rho}_{e}(0) \otimes \hat{\rho}_{b}(0)$. Only at that time would $z$ and $\eta$ be pure complex and Grassmannian numbers respectively. As the system evolves, both $\eta$ and $z$ contain Grassmann and c-number parts. The mixing of even and odd parts (note $g_{\mathbf{k}}$ is odd) comes about as the initially factorized atom state becomes "dressed".

We skip over the details of the derivation which can be found in the original paper [1] but simply present the master equation, here in operator form (at zero temperature with the field in a vacuum state) as follows: Writing

$$
\frac{\dot{u}(t)}{u(t)}=\Gamma(t)+i \Omega(t)
$$

the master equation reads

$$
\frac{\partial}{\partial t} \rho=-i[H(t), \rho]+\Gamma(t)\left\{S_{+} S_{-}, \rho\right\}-2 \Gamma(t) S_{-} \rho S_{+}
$$

where

$$
H(t)=\Omega(t) S_{+} S_{-}
$$

The first term corresponds to the unitary Hamiltonian evolution, only now the effect of the environment has induced a time dependent shift in the value

of the frequency, the second term is time dependent dissipation and the third corresponds to noise. 
The effect of the field is contained in the $u(s), \bar{u}(s)$ functions, which are obtained as solutions of the linear integro-differential equations

$$
\begin{aligned}
\dot{\eta}+i \omega \eta+\int_{0}^{s} d s^{\prime} \mu\left(s-s^{\prime}\right) \eta\left(s^{\prime}\right) & =0 \\
\dot{\bar{\eta}}^{\prime}-i \omega \bar{\eta}^{\prime}+\int_{0}^{s} d s^{\prime} \mu^{*}\left(s-s^{\prime}\right) \bar{\eta}^{\prime}\left(s^{\prime}\right) & =0
\end{aligned}
$$

under the condition

$$
u(0)=\bar{u}(t)=1
$$

Here, the kernel $\mu(s)$ is given by

$$
\mu(s)=\sum_{\mathbf{k}} g_{\mathbf{k}}^{2} e^{-i \omega_{\mathbf{k}} s}
$$

The equations for $u(t)$ functions can be solved with the use of the Laplace transform and the convolution theorem. They are given by

$$
u(s)=\mathcal{L}^{-1}\left(\frac{1}{z+i \omega+\tilde{\mu}(z)}\right)=\frac{1}{2 \pi i} \int_{c-i \infty}^{c+i \infty} \frac{d z e^{z s}}{z+i \omega+\tilde{\mu}(z)}
$$

where $\tilde{\mu}(z)$ is the Laplace transform of the kernel $\mu(s)$ and $c$ is a real constant larger than the real part of the poles of the integrand.

\subsection{Spontaneous emission}

To show how the standard results are regained, and to understand the meaning of the new function in the master equation, let us consider the physical

process of spontaneous emission. Start with a generic initial density matrix

$$
\rho=\left(\begin{array}{cc}
1-x & y \\
y^{*} & x
\end{array}\right)
$$

its corresponding Q-symbol is

$$
\rho(\bar{\eta}, \eta)=x+y^{*} \eta+y \bar{\eta}+(1-x) \bar{\eta} \eta
$$

If we evolve it with the density matrix propagator (derived in [1]) we obtain for the state at time $t$

$$
\rho_{t}(\bar{\eta}, \eta)=1-\bar{u} u(1-x)+\left(\bar{u} y^{*} \eta\right)+(u \bar{\eta} y)+(\bar{u} u(1-x)) \bar{\eta} \eta
$$


corresponding to

$$
\rho_{t}=\left(\begin{array}{cl}
\bar{u} u(1-x) & u y \\
\bar{u} y^{*} & 1-\bar{u} u(1-x)
\end{array}\right)
$$

Considering the case $x=y=0$ we get for the probability of spontaneous emission

$$
P(1 \rightarrow 0, t)=1-\bar{u} u
$$

The rate of decoherence in the energy eigenstates is governed by the absolute value of the function $u$ (the off- diagonal terms) while $u$ itself determines the rate of energy flow from the atom to the environment. Hence for our particular choice of initial state (vacuum) we find that decoherence and relaxation time are essentially identical . This equation is useful for studying decoherence of a qubit in a QED cavity.

\subsection{Field modes in Free Space and Cavity}

Our master equation (3.3) depends solely on the function $u(t)$, which in turn is determined by the kernel $\mu(s)$. In [1] we have given some analytic expressions for this function in various cases, including a single mode, an infinite number of modes in the field, and a cavity consisting of two parallel plates at distance $L$. The field satisfies Dirichlet boundary conditions on the surface of the plates. In all cases our results obtained from the generalized master equation bear closer resemblance qualitatively to the 2LA behavior than that of quantum Brownian motion. Details can be found in [1]. We now summarize the major findings obtained there.

\section{Discussion}

The physics of a $2 \mathrm{LA}-\mathrm{EMF}$ system at zero temperature is characterized by a number of time constants:

1) The inverse natural frequency $\omega_{0}^{-1}$

2) The inverse coupling constant $g_{\mathbf{k}}^{-1}=\sqrt{\omega_{\mathbf{k}}} / \lambda$

3) The relaxation time constant $\Gamma^{-1}$

4) The cavity size $L$ (divided by c)

First consider a zero temperature field in free space, thus ignoring factor 4). Start with only one mode in the field in resonance with the atom (whence 
we omit the subscript in $g_{\mathbf{k}}$ ), then the system undergoes Rabi nutation with frequency $\Omega \approx g \sqrt{n+1}$, where $n$ is the photon number in the field. The collapse time (assuming a large mean photon number $\bar{n}$ ) is $g^{-1}$, and revival time is $2 \pi \sqrt{\bar{n}} / g$. [2]. Atom excitation becomes significant in a time much greater than $\omega_{0}^{-1}$ but shorter than $g^{-1}$. (This is the condition for a first order perturbation theory to give reasonable results.) For a large number of modes, spontaneous emission occurs at the relaxation time scale $\Gamma^{-1}=\pi / g>>\omega_{0}^{-1}$ which we found to be the same as the decoherence time - the time for the off diagonal elements of the reduced density matrix to decay. When the mean number of photons in the field is large $(\bar{n}>>1)$, they become comparable to the collapse time. This is a measure of the coherence in the atom-field system, and is controlled mainly by their coupling and the photon number in the field. We see that with the resonance condition, the nature of decoherence in 2LS is very different from the QBM situation, where phase information in the Brownian particle is efficiently dispersed in the many modes in the bath coupled almost equally to the system. As we remarked in the Introduction, the identification of the phase information and energy flow from the 2LS to its environment is similar to the spin echo phenomena (Landau 'damping') which is based on statistical mixing rather than dissipation. The mathematical distinction lies between considering the system coupled to the continuous amplitude basis (QBM) of the environment or to the discrete number basis (2LA). The QBM case essentially produces noise that drives the system in a way insensitive to its own intrinsic dynamics. In our model, the coupling respects the internal dynamical structure of the 2LS and allows it to keep its coherence.

To see how the distribution of modes in a field changes the picture, the cavity field calculation is useful. There, as the plots in [1] show, the relaxation constant develops peaks and minima. The resonance effect is enhanced by a cavity size commensurate with the natural frequency of the 2LA and dissipation weakens. Narrow band resonance fluorescence as well as inhibition of spontaneous decay by frequent measurements - the Quantum Zeno effect - are interesting phenomena which our equations can provide finer details.

Non-Markovian processes involve memory effects (nonlocal in time). When the reaction time of the bath is comparable to or faster than the natural time scale of the system $\left(\omega_{0}\right)$, one also expects to see non-Markovian behavior. For the QBM problem, the case of high temperature Ohmic bath is almost the only condition that would yield a Markovian dynamics. For 
other types of spectral density (supraohmic) or for a low temperature bath, the dynamics of the system is generally non-Markovian [25]. By contrast, the 2LA is quite different: At zero temperature there is only one time scale $\Gamma^{-1}=\lambda^{-2} \omega^{-1}>>\omega^{-1}$ that determines both decoherence and relaxation. There is no memory effect and hence the process is Markovian. We expect that at finite temperature the dynamics of the 2LA will be nonMarkovian [39]. This is because there are more ways for the atom and the field to get entangled, and the memory effects of their interaction would presumably persist.

To conclude, we show that not all two level systems (2LS) decohere like quantum Brownian particles (QBM). Specifically, in the case of a two-level atom (2LA) interacting with an electromagnetic field (EMF) alone through the standard $\hat{\sigma}_{ \pm}$coupling as in a quantum optics / cavity QED quantum computer prototype, decoherence time of the $2 \mathrm{LA}$ is close to the relaxation time. This behavior is completely different from the QBM. The crucial difference lies in the type of coupling between the 2LS and the environment: The 2LA interacts resonately with selected modes in the electromagnetic field while the QBM is coupled to all modes in the harmonic oscillator bath. Only for those mechanisms which can be described by a $\hat{\sigma}_{z}$ type of coupling of the $2 \mathrm{LS}$ with the environment (which is subdominant in a 2LA-EMF system) will one expect a QBM-like decoherent behavior. Therefore one has to be careful in specifying what type of 2LS constitutes the qubit and and how it couples to the field, since the field can act as a resonator (2LA) or as a bath (QBM) producing very different decoherent behavior. In a realistic experiment one needs to know which processes are describable by what type of coupling and the weight of their relative contributions in varying environmental conditions before drawing credible conclusions about the overall nature and degree of decoherence in that system.

Acknowledgement This is a summary of work done with Dr. Charis Anastopoulos based on [1], and ongoing work with Adrian Dragulescu and Sanjiv Shresta, with whom I have enjoyed many useful discussions and fruitful collaboration. This work is supported in part by NSF grant PHY98-00967.

\section{References}


[1] C. Anastopoulos and B. L. Hu, Phys. Rev. A 62, (2000) 033821.

[2] D. F. Walls and G. J. Milburn, Quantum Optics ( Springer Verlag, Berlin, Heidelberg, 1994).

[3] L. Mandel and E. Wolf, Optical Coherence and Quantum Optics (Cambridge University Press, Cambridge, 1995).

[4] M. O. Scully and M. Suhail Zubairy, Quantum Optics (Cambridge University Press, Cambridge, 1997).

[5] M. Weissbluth, Photon-Atom Interactions (Academic Press, San Diego, 1988).

[6] W. Vogel and D. G. Welsch, Lectures on Quantum Optics, (Akademie Verlag, Berlin, 1994).

[7] G. Compagno, R. Passante and F. Persico, Atom-Field Interactions and Dressed Atom (Cambridge University, Cambridge, 1995).

[8] H. J. Carmichael, An Open Systems Approach to Quantum Optics (Springer Verlag, Berlin, 1993).

[9] A. O. Caldeira and A. J. Leggett, Ann. Phys. (NY) 149, 374 (1983). A. J. Leggett et al., Rev. Mod. Phys. 59, 1 (1987).

[10] U. Weiss, Quantum Dissipative Systems (World Scientific, Singapore, 1993).

[11] I. L. Chuang et al, Science 270, 1633 (1995. D. P. DiVencenzo et al, Science 270, 255 (1995). P. W. Shor, Phys. Rev. A52, R2493 (1995). J. I. Cirac and P. Zoller, Phys. Rev. Lett. 74, 4091 (1995). A. Ekert and R. Jozsa, Rev. Mod. Phys. 68, 733 (1996). Also the excellent book by M. A. Nielsen and I. L. Chuang, Quantum Computation and Quantum Information (Cambridge University Press, Cambridge, 2000)

[12] W. G. Unruh, Phys. Rev. A51, 992 (1995).

[13] G. M. Palma, K.-A. Suominen and A. K. Ekert, Proc. Roy. Soc. London A 452, 567 (1996). 
[14] P. Zanardi and M. Rasetti, Phys. Rev. Lett. 79, 3306 (1997).

[15] M. B. Plenio and P. L. Knight, Proc. Rey. Soc. Lon. A453, 2017 (1997).

[16] S. Schneider and G. J. Milburn, quant-ph/9710044 (1997).

[17] L. Viola and S. Lloyd, Phys. Rev. A58, 2733 (1998).

[18] A. Garg, quant-ph/9803071 (1998).

[19] P. Zanardi, Phys. Rev. A57, 3276 (1998).

[20] See, e.g., E. Joos and H. D. Zeh, Z. Phys. B59, 223 (1985); D. Giulini et al, Decoherence and the Appearance of a Classical World in Quantum Theory (Springer Verlag, Berlin, 1996). W. H. Zurek, Physics Today 44, 36 (1991); Prog. Theor. Phys. 89, 281 (1993). J. P. Paz and W. H. Zurek, "Environment-Induced Decoherence and the Transition From Quantum to Classical" 72nd Les Houches Summer School on "Coherent Matter Waves", July-August 1999. quant-ph/0010011

[21] R. P. Feynman and A. R. Hibbs, Quantum Mechanics and path integrals (McGraw-Hill, New York, 1965) ; R. P. Feynman and F. L. Vernon, Ann. Phys. (N. Y.) 24, 118 (1963).

[22] A. O. Caldeira and A. J. Leggett, Physica A 121, 587 (1983). Phys. Rev. A31, 1059 (1985).

[23] H. Grabert, P. Schramm and G. L. Ingold, Phys. Rep. 168, 115 (1988).

[24] W. G. Unruh and W. H. Zurek, Phys. Rev. 40, 1071 (1989).

[25] B. L. Hu, H. P. Paz and Y. Zhang, Phys. Rev. D45, 2843 (1992); Phys. Rev. D47, 1576 (1993).

[26] B. L. Hu and A. Matacz, Phys. Rev. D 49, 6612 (1994).

[27] J.J.Halliwell, T.Yu, Phys. Rev. D53 (1996) 2012-2019

[28] G. W. Ford, R. F. O'Connell, Phys. Rev. D64 (2001)105020

[29] J. P. Paz, S. Habib and W. H. Zurek, Phys. Rev. 47, 488 (1993). 
[30] W. H. Zurek, S. Habib and J. P. Paz, Phy. Rev. Lett. 70, 1187 (1993)

[31] B. L. Hu and Y. Zhang, Mod. Phys. Lett. A8, 3575 (1993), Int. J. Mod. Phys. 10, 4537 (1995).

[32] A. Anderson and J. J. Halliwell, Phys. Rev. D48, 2753 (1993); C. Anastopoulos and J. J. Halliwell, Phys. Rev. D51, 6870 (1995); C. Anastopoulos, Phys. Rev. E53, 4711 (1996).

[33] J. P. Paz and W. H. Zurek, "Environment-Induced Decoherence and the Transition From Quantum to Classical" 72nd Les Houches Summer School on "Coherent Matter Waves", July-August 1999. quant$\mathrm{ph} / 0010011$

[34] J. R. Anglin, R. Laflamme, W. H. Zurek and J. P. Paz, Phys. Rev. D52, 2221 (1995).

[35] B. L. Hu and D. Pavon, Phys. Lett. B180, 329 (1986); H. E. Kandrup, Phys. Rev. D37, 3505 (1988); D38, 1773 (1988).

[36] S. Habib, Y. Kluger, E. Mottola and J. P. Paz, Phys. Rev. Lett. 76, 4660 (1996)

[37] S. K. Ma, Statistical Mechanics (World Scientific, Singapore, 1995)

[38] P. M. V. Barone and A. O. Caldeira, Phys. Rev. A 43, 57 (1991).

[39] Charis Anastopoulos, Adrian Dragulescu, B. L. Hu and Sanjiv Shresta, "Two-Level Atom-Field Interaction at Finite Temperatures" 